\title{
Temporal Solitonic Crystals and Non-Hermitian Informational Lattices
}

\author{
Jaroslaw E. Prilepsky, ${ }^{1, *}$ Stanislav A. Derevyanko, ${ }^{1}$ and Sergei K. Turitsyn ${ }^{2}$ \\ ${ }^{1}$ Nonlinearity and Complexity Research Group, Aston University, B4 7ET Birmingham, United Kingdom \\ ${ }^{2}$ Photonics Research Group, Aston University, B4 7ET Birmingham, United Kingdom
}

(Received 21 November 2011; published 4 May 2012)

\begin{abstract}
Clusters of temporal optical solitons - stable self-localized light pulses preserving their form during propagation-exhibit properties characteristic of that encountered in crystals. Here, we introduce the concept of temporal solitonic information crystals formed by the lattices of optical pulses with variable phases. The proposed general idea offers new approaches to optical coherent transmission technology and can be generalized to dispersion-managed and dissipative solitons as well as scaled to a variety of physical platforms from fiber optics to silicon chips. We discuss the key properties of such dynamic temporal crystals that mathematically correspond to non-Hermitian lattices and examine the types of collective mode instabilities determining the lifetime of the soliton train. This transfer of techniques and concepts from solid state physics to information theory promises a new outlook on information storage and transmission.
\end{abstract}

PACS numbers: 42.81.Dp, 45.05.+x, 61.72.-y, 89.70.-a

The concept of a soliton is one of the most unifying theories of modern physics with nonlinear solitary waves observed and studied in a range of diverse fields of science (see, e.g., [1-6]). The term "soliton" implies stable robust structures that can propagate in time or space without changing their shape. Stability makes solitons very attractive building blocks for numerous theories, experimental techniques, and practical applications. Chains of solitons have been studied, mostly in the context of the mathematical inverse scattering transform applied to the Toda chain model [7-11], though the resulting equations are quite universal [10]. We suggest here a rather different concept that goes far beyond previously studied problems. The key novel point is that we propose the concept of dynamical lattices of solitons with different phases used for carrying or storing information, thus forming temporal solitonic information crystals (SICs). We refer to the soliton train as the solitonic crystal underlining the similarities with the theory of crystal lattices, though the interpretation of the results applies directly to the information transmission. Controlled variation of soliton phases is principally important, because it makes it possible to encode, transmit, and store information using soliton dynamical lattices. As we shall see, the proposed concept provides an interesting link between the theory of crystal lattice, nonlinear science, photonics, and information theory.

The properties of soliton "crystallization" depend on the number $M$ of the phase levels allowed for each soliton from the cluster. This number is also a number of states that can be used for coding information-it determines a data

Published by the American Physical Society under the terms of the Creative Commons Attribution 3.0 License. Further distribution of this work must maintain attribution to the author(s) and the published article's title, journal citation, and DOI. modulation format $[12,13]$. To illustrate the proposed idea, without loss of generality, we study the dynamical properties of the particular information crystal formed by pulses with phases belonging to the discrete set $\{2 \pi b / M\}_{b=0}^{M-1}$. Each of these phases (or phase differences) can be linked to a symbol with an integer value: $b=0,1, \ldots, M-1$. In communication applications, such a choice of phases corresponds to a very practical and important $M$-level phaseshift keying ( $M$-PSK) data format $[12,13]$.

Compared to conventional crystals encountered in condensed matter physics, the SICs have more freedom in the design parameters that determine their properties. The first important problem with regard to the considered objects is their lifetime, which, obviously, depends on the "crystal design," i.e., on the information encoded into the sequence of soliton pulses. We study here the lifetime of soliton trains (SICs) defined through the instabilities in the pulse train leading to the corruption of the information stream. Such instabilities are caused by the nonlinear interaction of the lattice elements (solitons). To illustrate our idea, we consider here a specific example of a train formed by a chain of optical solitons of the nonlinear Schrödinger equation. In the practical limit of well separated solitonic "atoms," the dynamical problem is reduced to the complex Toda chain equations [7-9,11], which, under realistic assumptions (exactly matching the requirements for the information transmission), describe the evolution of the position and phase for each individual soliton of the train. We investigate how the information content of the pattern affects the train dynamics and elucidate general mechanisms for the appearance of unstable collective modes that define the lifetime of the SIC. This lifetime is directly relevant to applications in telecommunications and information theory. The collective motion of the SIC is tantamount to the dynamics of a non-Hermitian linear lattice under an external force, and the growth increments of the 
ensuing modes define its lifetime. Using the similarity between conventional crystal lattice dynamics in the presence of localized defects and the proposed "informational lattices," one can apply the well developed methods of condensed matter physics [6] to the description of information coding and distortions. The introduction of new data coding formats with particular predefined properties can be based on the studies of the dynamical properties of the encoded train: The knowledge of the SIC eigenmode structure can then be used for the composition of new formats with a given lifetime.

We emphasize that, for coherent optical communication, the proposed technique can offer an interesting alternative to quasilinear transmission with uncompensated dispersion and subsequent digital signal processing [14,15]. Uncompensated dispersion broadens optical pulses during the propagation over a very large number of time slots, thus introducing strong patterning effects and making compensation of nonlinear effects quite a challenging problem [16-18]. The use of multilevel modulation formats in coherent transmission allows one to decrease the symbol (baud) rate [12], i.e., the speed at which each symbol is transmitted while keeping the total effective rate very high. For multilevel data coding using $M$ levels with large enough $M$, the gain in the information rate is $\log _{2}(M)$, which makes possible the transmission of symbols at relatively low (for optical communication) rates of, say, $1 \mathrm{GHz}$. Evidently, the larger $M$, the smaller the rate that can be used. In this regime the width of an optical soliton in a standard single mode telecommunication fiber can be of the order of 200 ps (and broader) with the average signal power of the scale of only $0.1 \mathrm{~mW}$ per channel. Such a small signal power is still sufficient to provide for a continuous balance between the Kerr nonlinearity and the fiber dispersion to form a soliton and to take advantage of dispersion uncompensated transmission. In the proposed soliton-based coherent communication approach, a large part of nonlinear distortions is compensated by the dispersion removing the need for separate compensation of those two factors, similar to the previously studied on-off-keying direct detection soliton transmission technique (without use of an optical phase for information coding) [3,4]. The novel point here is the context of coherent transmission and hence the possibility to undo the remaining nonlinear impairments through nonlinear digital signal processing. This paves the way to go well beyond the current capacity limitations of traditional optical communications [13-15,19,20]. The analytical knowledge of SIC properties allows one to apply efficient digital signal processing to mitigate the effects of the nonlinear interaction. We would like to stress once more that optical fiber communication is just one particular example of the application of the proposed general concept of information crystals.

Without loss of generality, we consider here as the master model the nonlinear Schrödinger equation with constant anomalous dispersion:

$$
\frac{\partial Q}{\partial Z}-\frac{i}{2} \frac{\partial^{2} Q}{\partial t^{2}}-i|Q|^{2} Q=0,
$$

written here in soliton units; see Refs. [3-5,21] for particular values of normalizing parameters: $Q(t ; Z)$ is the optical pulse envelope, $Z$ stands for the propagation distance, and $t$ is the time in the frame comoving with the group velocity of the envelope. The proposed information crystal is built from a train of equidistant same amplitude fundamental solitons of Eq. (1) [2] with different phases (used for carrying information): $Q(t ; 0)=\sum_{n=0}^{N} 2 \nu e^{i \phi_{n}(t)} /$ $\cosh \left[2 \nu\left(t-\xi_{n}\right)\right]$, where $\phi_{n}$ and $\xi_{n}$ are, respectively, the phase and position of the $n$th individual soliton and $2 \nu$ is the amplitude, $N+1$ being the total number of solitons in the train. The exponential overlap between the adjacent solitons, $\varepsilon=\exp \left[-\nu\left(\xi_{n+1}-\xi_{n}\right)\right]$, is assumed to be small; i.e., the initial intersoliton separation is much larger that the soliton width $\sim \nu^{-1}$. For the description of the dynamics of soliton positions $\xi_{n}$ and phases $\phi_{n}$, we introduce a complex variable $q_{n}$ as $q_{n}(Z)=-2 \nu \xi_{n}(Z)+2 n \ln 2 \nu+$ $i\left[n \pi-\phi_{n}(Z)\right]$ with the $Z$ dependence of the soliton phase $\phi_{n}(Z)=2 Z \nu^{2}+\phi_{n}(0)$ in the case of the noninteracting pulses. The initial phase values $\phi_{n}(0)$ (or rather their differences) carry the information content to be transmitted: $\Delta \phi_{n}(0)=2 \pi b_{n} / M$, with the first difference operator defined as $\Delta \phi_{n} \equiv \phi_{n+1}-\phi_{n}$. The initial stage of the train dynamics (in the first order in $\varepsilon$ ) can be described by means of the complex Toda chain model [7-9,11]: $\partial_{Z Z} q_{n}=16 \nu^{2}\left[e^{\Delta q_{n}}+e^{\Delta q_{n-1}}\right]$; see [22].

We assume that initially all solitons are equidistant with the common distance $r=\xi_{n+1}(0)-\xi_{n}(0)$. We now linearize the Toda model around the initial configuration $q^{0}$ [11]: $q_{n}(Z)=q_{n}(0)+w_{n}(Z),\left|w_{n}\right| \ll\left|\Delta q_{n}(0)\right|$. The initial differences contain the encoded information: $\Delta q_{n}(0)=-2 \nu r+2 \ln 2 \nu+i\left[\pi-\Delta \phi_{n}(0)\right] . \quad$ Furthermore, we rescale the propagation distance $Z$ as $8 \nu^{2} e^{-r \nu} Z \rightarrow z$. After the linearization, we arrive at a set of inhomogeneous equations of the form

$$
\frac{d^{2} \mathbf{w}}{d z^{2}}=\hat{\Lambda} \mathbf{w}+\mathbf{f}
$$

where $\hat{\Lambda}$ is a tridiagonal complex $(N+1) \times(N+1)$ matrix. By introducing symbol indicator quantities $\sigma_{n}=$ $\exp \left[i \pi\left(M-2 b_{n}\right) / M\right]$, the elements of the coupling matrix are expressed as $\Lambda_{n n}=-\left(\sigma_{n}+\sigma_{n+1}\right), \Lambda_{n n-1}=\sigma_{n}$, and $\Lambda_{n n+1}=\sigma_{n+1}$, while the components of the driving vector f are $f_{n}=\Delta \sigma_{n-1}$. In Eqs. (2), the initial $w_{n}(0)$ and their $z$ derivatives can be set to zero.

The solution of Eq. (2) can be written as $w_{n}(z)=$ $\int_{0}^{z} d \tau f_{m} G_{n m}(z-\tau)$, where the Laplace transform $\hat{G}(\gamma)$ of the matrix Green function $\hat{G}$ corresponds to the resolvent of $\hat{\Lambda},(\lambda-\hat{\Lambda})^{-1}$, evaluated at the argument $\lambda=-\gamma^{2}$. Each pole of the Green function, $\lambda_{k}$, corresponds to an eigenvalue of the matrix $\Lambda$ and to an eigenmode of system Eq. (2) growing with distance as $\gamma_{k}^{-1} \sinh \gamma_{k} z, \gamma_{k}=\lambda_{k}^{1 / 2}$. 

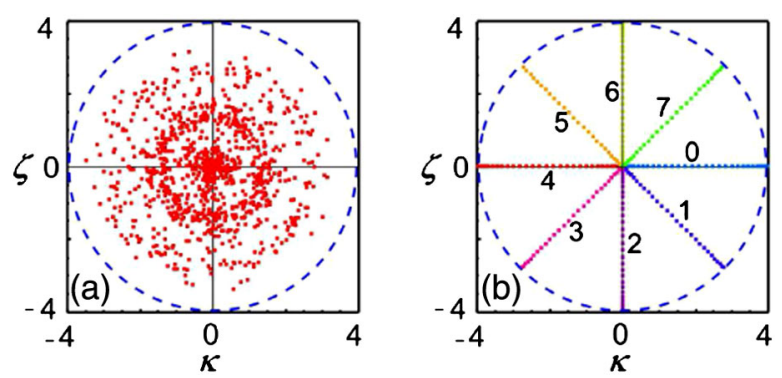

FIG. 1 (color online). Eigenvalue distributions for the 8-PSK format in the complex plane of $\lambda=\kappa+i \zeta$. Pane (a) shows the typical eigenvalue distribution for a completely random pattern. Dashed circles mark the theoretical spectrum boundary: $|\lambda|=4$. Pane (b) shows the spectra for all possible homogeneous patterns: Each colored ray from the origin corresponds to a particular value of symbol $b$.

For any transmitted pattern the locus of eigenvalues is $\left|\lambda_{k}\right| \leq 4$ (dashed circle in Fig. 1). Thus, the quantity $\operatorname{Re}\left[\gamma_{k}\right] \leq 2$ defines the growth increment of each eigenmode, and the characteristic lifetime of the SIC is given by the eigenvalue corresponding to the largest positive real part: $\max _{k} \operatorname{Re}\left[\gamma_{k}\right]$. The typical pattern of $\lambda_{k}$ for the SIC corresponding to a random input 8-PSK is given in Fig. 1(a).

For the uniform inputs (all symbols are the same, $b_{n}=$ $b)$, we have the common symbol indicator $\sigma_{b}=$ $\exp [i \pi(M-2 b) / M]$, and the expression for the spectrum is $\lambda_{k}=-2 \sigma_{b}\left(1-\cos \rho_{k}\right)$ with $\rho_{k}=\pi k /(N+1)$; see Fig. 1(b). For $b=M / 2$, the spectrum coincides with that of the classical phonon dynamics [6], and this pattern is stable. We will mark with the index " 0 " the coupling matrix which corresponds to this stable case: $\hat{\Lambda}^{0}$.

The direct calculation of the resolvent for the homogeneous pattern can be performed analytically by taking into account that the number of symbols, $N$, is very large. Then for the stable configuration $b=M / 2$ one arrives at

$$
G_{m n}^{0}(\lambda)=\frac{1}{\sqrt{4 \lambda+\lambda^{2}}}\left[F(\lambda)^{n+m+1}+F(\lambda)^{|n-m|}\right],
$$

where $2 F(\lambda)=\lambda+2-\left(4 \lambda+\lambda^{2}\right)^{1 / 2}$. The use of Eq. (3) assumes that we deal with a semi-infinite system, where the site positions $n$ and $m$ are counted from its edge; the method of calculation is similar to that used in the theory of crystal lattices [6].

To demonstrate further the analogy with crystal lattice theory, let us consider isolated "defects" embedded in the homogeneous SIC. Consider a single "defect symbol" $b_{s}=b$ in an otherwise stable pattern comprising only $b_{n}=M / 2$. This means that the phase difference between $(s+1)$ th and $s$ th solitons $\Delta \phi_{s} \neq \pi$, whereas the remaining differences are equal to $\pi$. The resulting perturbation $\hat{P}$ of matrix $\hat{\Lambda}$ is local: $\hat{\Lambda}=\hat{\Lambda}^{0}+\hat{P}$, where $\hat{P}$ is determined by the value of the defect symbol indicator $\sigma_{b}: P_{s s}=$ $P_{s+1 s+1}=1-\sigma_{b} ; \quad P_{s s+1}=P_{s+1 s}=-P_{s s} \quad$ (the other elements are 0); Eqs. (2) remain the same, except for those sites with numbers $s$ and $s+1$. The nonzero components of the driving vector are $f_{s}=\sigma_{b}-1$ and $f_{s+1}=-f_{s}$.

The perturbed set of dynamical equations is structurally similar to equations for the crystal lattice vibrations with a single defect bond [6]. A significant difference from the classical theory is that the resulting defect bond is imaginary, and the perturbed matrix $\Lambda$ is generally nonHermitian. In the case of the defect symbol value $b=0$, matrix $\hat{\Lambda}$ remains Hermitian but not negatively definite: This means that the defect bond is nonelastic as well. So, for any value of the defect symbol $b \neq M / 2$, one should expect the appearance of unstable defect modes.

We now examine the localized eigenvalue which produces this instability. Again one can use ideas similar to those of the classical theory of crystal lattice defects [6] even though the site-coupling matrix $\hat{\Lambda}$ is a non-Hermitian one. To define the defect eigenvalue, one needs to calculate the resolvent corresponding to that perturbed matrix, which can be done explicitly by utilizing the Woodbury formula [23] for perturbed inverse matrices. The latter is a generalization of the approach first used by Lifshitz [24] for determining the frequencies of localized impurity modes in an otherwise perfect crystal lattice (see also [6]). Our initial unperturbed matrix $\hat{\Lambda}^{0}$ corresponds to a homogeneous pattern with $b_{n} \equiv M / 2, \sigma_{n} \equiv 1$. Then we seek for the spectrum of the perturbed one, $\hat{\Lambda}=\hat{\Lambda}^{0}+\hat{P}$. If the defect symbol is settled far from the system edges, the localized eigenvalue becomes almost independent of $s$, and the expression for the localized eigenvalue is [see Fig. 2(a) for the spectrum of 8-PSK]

$$
\lambda_{b}=\frac{4 \sigma_{b}^{2}}{1-2 \sigma_{b}}+O\left(e^{-s}\right)
$$

Because the perturbation is localized on a single bond between the two sites (solitons), the corresponding unstable defect mode is localized on this bond as well; see Figs. 2(c)-2(f). This is similar to phonon modes in a crystal lattice with a defect [6]. The expression for the width of the localized mode corresponding to the defect $b$ is

$$
R_{b} \approx-\left.\ln ^{-1}|F(\lambda)|\right|_{\lambda=\lambda_{b}} .
$$

If the defects are sparse and do not interact, the localized eigenvectors do not "feel" the presence of other neighboring defects, and the typical spectrum of $\lambda_{k}$ consists of the stable band at the real negative axis (located between -4 and 0), and the "defect speckles" in the complex plane: These will be densely concentrated in the vicinity of points defined by Eq. (4), as seen in Fig. 2(a) for a typical pattern of the noninteracting defects in a 8-PSK symbol train. To satisfy the sparseness condition, the concentration of the defect symbols, $c$, must satisfy $c<c_{b}=\left(2 R_{b}\right)^{-1}$. The values of $R_{b}$ can be found 

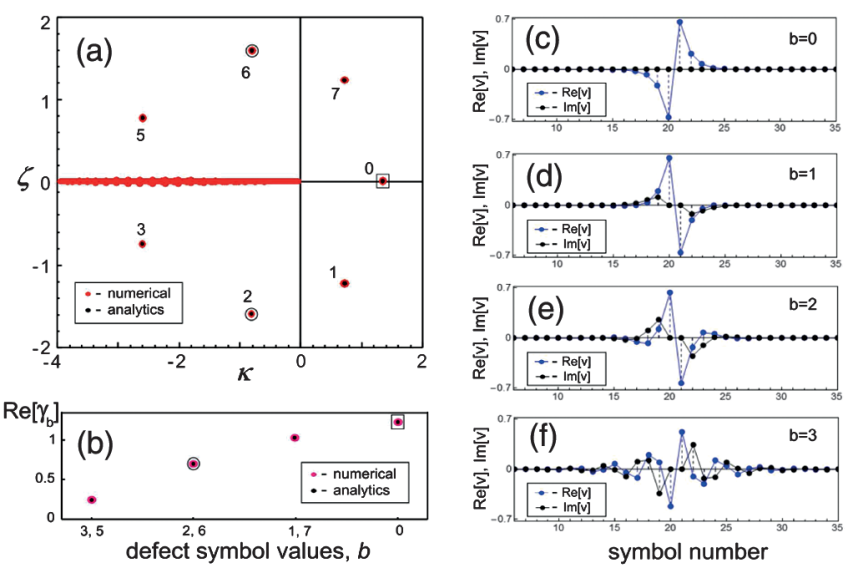

FIG. 2 (color). Pane (a): The spectrum of a 8-PSK pattern where each 7th symbol is randomly coded. The digits near the localized eigenvalues refer to the value of $b$ for an isolated defect. Circles mark the common eigenvalues with the 4-PSK format, and the square indicates the defect eigenvalue for the $b=0$ symbol, which is present in all $M$-PSK formats. The analytical values are defined via Eq. (4). Pane (b): The increment values $\operatorname{Re}\left[\gamma_{b}\right]$ for the defect modes. Panes (c)-(f) give the profiles of localized eigenvectors, corresponding to isolated defect symbols.

via Eq. (5), and the results for 2-, 4-, and 8-PSK are summarized in Table I: The mode width grows as one moves away from the most unstable mode with $b=0$ (with $\lambda_{0}=4 / 3$ ); Figs. 2(c)-2(f). Note that the width of the most unstable mode, $R_{0} \approx 0.91$, is the lowest possible for any PSK format.

We would like to stress that the lifetime of a dynamic crystal can be to some extent (limitations are imposed by a single pulse transmission) controlled by the crystal design, first of all, by separation of the "atoms" (pulses) from each other. For standard communication systems where transmission distance is always limited, it is possible to design the information crystal in such a way that its lifetime (distance at which instability is developed) will always be much larger than the transmission distance. In addition, coding can be used to remove the patterns that correspond to the maximum instability [25]. While the increase of the distance between pulses is the major way to increase lifetime, the instability can be also controlled by using, e.g., the sparse encoding format (SEF) based on utilization of a stable uniform pattern corresponding to $b=M / 2$ as a matrix or informational substrate into which we sparsely

TABLE I. Values of the $R_{b}$ as given by Eq. (5) and corresponding critical concentrations $c_{b}$.

\begin{tabular}{lcc}
\hline \hline$M, b$ & $R_{b}$ & $c_{b}$ \\
\hline Any $M, b=0$ & 0.91 & 0.55 \\
$M=8, b=1,7$ & 0.97 & 0.51 \\
$M=8, b=2,6$, or $M=4, b=1,3$ & 1.24 & 0.40 \\
$M=8, b=3,5$ & 2.58 & 0.19 \\
\hline \hline
\end{tabular}

embed the information-carrying symbols (with the spacings exceeding $2 R_{b}$; see the Supplemental Material [21] for the emergence of highly unstable modes for dense coding). The information can be carried by both the phase of the substrate and the embedded symbol. The alphabet of such a format can be large enough while instability will be substantially suppressed. The measure of "sparseness" is determined by the lowest value of $c_{b}$ : For the 8-PSK, this means $c<c_{3,5}$. Each defect symbol would bring about the appearance of the localized growing eigenmode. Again for the 8-PSK case this regime is achieved for interdefect distance $R>\operatorname{int}\left[2 R_{3,5}\right] \approx 6$; see Table I. The growth rate of the corresponding mode depends only on the value of a corresponding defect symbol. Since we know exactly the growth rate $\operatorname{Re}\left[\gamma_{b}\right]$ for each defect mode, one can readily estimate the typical lifetime of the encoded pattern; see Fig. 2(b): The maximal instability increment corresponds to $\lambda_{0} \approx 4 / 3$, for which we have $\gamma_{0}=2 / \sqrt{3}$. For other defect modes with $b \neq 0$, this value is lower. Compare this to general patterns [see Fig. 1(a)] where the instability rate is $\gamma \approx 2$. Therefore by applying this embedding procedure (i.e., using SEF) we can increase the lifetime of a pulse train and hence the maximum error-free propagation distance compared to conventional dense encoding. Note that the usage of more sophisticated (compared to SEF) input encoding, like, e.g., correlated input, can enhance the overall bit rate of the transmission while one keeps the lifetime of a SIC at the same level.

Figure 3 shows the results of the direct numerical modeling of Eq. (1) illustrating the increased stability of the SEF compared to arbitrary symbol patterns. The evolution of relative phase differences, $\delta \phi_{n}(z) /\left(2 \pi M^{-1}\right)$ (phase jitter), is shown for a completely random initial pattern [Fig. 3(a)] and sparsely encoded 8-PSK input with $R=$ $7>2 R_{3,5}$ [Fig. 3(b)]. It is seen that the implementation of the SEF reduces phase jitter by more than $50 \%$. We would like to stress once more that the lifetime of SIC defined as the inverse increment of amplitude growth can be controlled by appropriate design and dramatically enhanced by increasing the distance $R$ between the encoded symbols beyond the extent of localized unstable modes defined by Eq. (5). The Supplemental Material [21] also contains the corresponding results for the timing jitter.

In conclusion, in this Letter, we have introduced the concept of informational crystals formed by lattices of temporal pulses (solitons) with varying phases. The phases of individual pulses are used to carry the information content. Within the approach proposed, we elucidated the mechanisms of the appearance and development of instabilities. The instabilities in the initial evolution are always present in the linear dynamics as the coupling matrix for the ensuing linear system for phase and position deviations is generally non-Hermitian and subject to an "external force." We have proposed to use such solitonic information crystals with solitons being transmitted at relatively low 

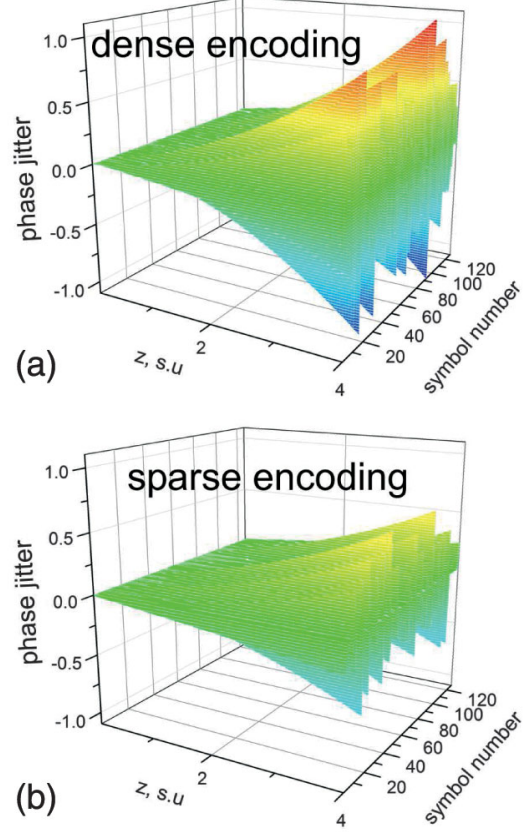

FIG. 3 (color). Phase jitter (relative phase deviations) dynamics for the 8-PSK encoding scheme obtained by the direct integration of Eq. (1) for a dense encoding format (a) and the SEF input where only each 7 th symbol is used for coding (b). Two pseudorandom sequences were used for each case. The distance $Z$ is measured in soliton units, s.u.

rates allowing us to provide a continuous balance between the fiber nonlinearity and dispersion effects for coherent transmission systems to achieve communication capacity well beyond the limits of traditional (quasilinear) optical communication techniques.

The knowledge of instability spectra [like, e.g., the one shown in Fig. 1(a)] allows one to identify the most "dangerous" patterns [21], which can then be removed by using data coding or by other techniques that can substantially reduce the frequency of their appearance [25] to achieve maximum stability of the soliton crystal. Sparse encoding is an additional possibility to increase crystal lifetime (although not necessarily the optimal one). What makes the sparse encoding interesting, however, is that we have been able to utilize the machinery of the theory of crystal lattice defects-which again illustrates the potential for cross-fertilization of the two fields. We believe that the proposed concept of temporal information crystals and the transfer of techniques from condensed matter physics to the area of telecommunications and information theory might lead to a completely new outlook on information storage and transmission.

*y.prylepskiy1@aston.ac.uk

[1] A.C. Newell, Solitons in Mathematics and Physics (SIAM, Philadelphia, 1985).
[2] V.E. Zakharov, S. V. Manakov, S. P. Novikov, and L.P. Pitaevskii, Theory of Solitons. The Inverse Scattering Method (Consultants Bureau, New York, 1984).

[3] A. Hasegawa and Y. Kodama, Solitons in Optical Communications (Oxford University, Oxford, 1995).

[4] L.F. Mollenauer and J.P. Gordon, Solitons in Optical Fibers: Fundamentals and Applications (Academic, San Diego, 2006).

[5] Yu. S. Kivshar and G. P. Agrawal, Optical Solitons: From Fibers to Photonic Crystals (Academic, San Diego, 2003).

[6] A. M. Kosevich, The Crystal Lattice: Phonons, Solitons, Dislocations (Wiley-VCH, Berlin, 1999).

[7] V.S. Gerdjikov, D. J. Kaup, I. M. Uzunov, and E. G. Evstatiev, Phys. Rev. Lett. 77, 3943 (1996).

[8] V.S. Gerdjikov, I. M. Uzunov, E. G. Evstatiev, and G. L. Diankov, Phys. Rev. E 55, 6039 (1997).

[9] V. S. Gerdjikov, E. G. Evstatiev, D. J. Kaup, G. L. Diankov, and I. M. Uzunov, Phys. Lett. A 241, 323 (1998).

[10] V. S. Gerdjikov, E. V. Doktorov, and J. Yang, Phys. Rev. E 64, 056617 (2001).

[11] J. M. Arnold, J. Opt. Soc. Am. A 15, 1450 (1998); Phys. Rev. E 60, 979 (1999).

[12] J. G. Proakis, Digital Communications (McGraw-Hill, New York, 2000).

[13] A. H. Gnauk and P. J. Winzer, J. Lightwave Technol. 23, 115 (2005); R.-J. Essiambre, G. J. Foschini, G. Kramer, and P. J. Winzer, Phys. Rev. Lett. 101, 163901 (2008).

[14] S. J. Savory, IEEE J. Sel. Top. Quantum Electron. 16, 1164 (2010).

[15] R. I. Killey and C. Behrens, J. Mod. Opt. 58, 1 (2011).

[16] A. Mecozzi, C. B. Clausen, and M. Shtaif, IEEE Photonics Technol. Lett. 12, 392 (2000).

[17] M. J. Ablowitz and T. Hirooka, IEEE J. Sel. Top. Quantum Electron. 8, 603 (2002).

[18] S. Vergeles and S. K. Turitsyn, Phys. Rev. A 83, 061801 (R) (2011).

[19] D. J. Richardson, Science 330, 327 (2010).

[20] A. D. Ellis and J. Zhao, in Impact of Nonlinearities on Fiber Optic Communications, edited by S. Kumar (Springer, New York, 2011), Chap. 13, p. 507.

[21] See Supplemental Material at http://link.aps.org/ supplemental/10.1103/PhysRevLett.108.183902 for the normalization and scale values recalculated in the real world units, explanations for the appearance of the fastgrowing modes, and the additional simulations referring to timing jitter.

[22] Under the assumptions of equal amplitudes and velocities for initial solitons $(\varepsilon \ll 1$ is assumed as well), the variations of the soliton amplitude and velocity are much slower than those for phases and positions and, thus, can be disregarded; see Ref. [7] and especially Ref. [8].

[23] M. Woodbury Memorandum, Statistical Research Group, Princeton University, Report No. 42, 1950 (unpublished); see the exhaustive survey in W. W. Hager, SIAM Rev. 31, 221 (1989).

[24] I. M. Lifshitz, Zh. Eksp. Teor. Fiz. 17, 1076 (1947).[Sov. Phys. JETP 17, 1076 (1947)].

[25] A. Shafarenko, A. Skidin, and S. K. Turitsyn, IEEE Trans. Commun. 58, 2845 (2010); A. Shafarenko, K. S. Turitsyn, and S. K. Turitsyn, ibid. 55, 237 (2007). 